Portland State University

PDXScholar

\title{
Approximate Equations of State in Two-Temperature Plasma Mixtures
}

John D. Ramshaw

Portland State University, jdramshaw@yahoo.com

Andrew W. Cook

Lawrence Livermore National Laboratory

Follow this and additional works at: https://pdxscholar.library.pdx.edu/phy_fac

Part of the Physics Commons

Let us know how access to this document benefits you.

\section{Citation Details}

Ramshaw, J. and Cook, A. (2014). Physics of Plasmas 21, 022706.

This Article is brought to you for free and open access. It has been accepted for inclusion in Physics Faculty Publications and Presentations by an authorized administrator of PDXScholar. Please contact us if we can make this document more accessible: pdxscholar@pdx.edu. 


\title{
Approximate equations of state in two-temperature plasma mixtures
}

\author{
John D. Ramshaw ${ }^{1,2}$ and Andrew W. Cook ${ }^{1}$ \\ ${ }^{1}$ Lawrence Livermore National Laboratory, Livermore, California 94551, USA \\ ${ }^{2}$ Department of Physics, Portland State University, Portland, Oregon 97207, USA
}

(Received 5 November 2013; accepted 6 February 2014; published online 20 February 2014; publisher error corrected 24 February 2014)

\begin{abstract}
Approximate thermodynamic state relations for multicomponent atomic and molecular gas mixtures are often constructed by artificially partitioning the mixture into its constituent materials and requiring the separated materials to be in temperature and pressure equilibrium. Iterative numerical algorithms have been employed to enforce this equilibration and compute the resulting approximate state relations in single-temperature mixtures. In partially ionized gas mixtures, there is both theoretical and empirical evidence that equilibrating the chemical potentials, number densities, or partial pressures of the free electrons is likely to produce more accurate results than equilibrating the total pressures. Moreover, in many situations of practical interest the free electrons and heavy particles have different temperatures. In this paper, we present a generalized algorithm for equilibrating the heavy-particle and electron temperatures and a third user-specified independent thermodynamic variable in a two-temperature plasma mixture. Test calculations based on the equilibration of total pressure vs. electron pressure are presented for three different mixtures. (C) 2014 AIP Publishing LLC. [http://dx.doi.org/10.1063/1.4866149]
\end{abstract}

\section{INTRODUCTION}

Multicomponent hydrodynamics calculations require accurate thermodynamic state relations for material mixtures. Unfortunately, constructing such relations for atomic or molecular mixtures of interacting materials is so difficult and laborious that it is rarely feasible to employ them in practical calculations. In practice, the state relations of the mixture must perforce be approximated in terms of those of the pure materials of which it is composed. An obvious and straightforward way of constructing such approximations is to regard the mixture as being artificially partitioned or separated into its constituent components or materials, so that each species or material $k$ occupies its own compartment or subvolume and possesses some initially undetermined portions of the specific volume and internal energies of the mixture. Different methods of apportioning or distributing the mixture volume and energy among its constituents lead to different approximations. The most common procedure (e.g., Refs. 1 and 2) has been to apportion the mixture volume and energy in such a way that the individual materials are in temperature and pressure equilibrium with one another, and to interpret the resulting equilibrated values thereof as the temperature and pressure of the mixture. This procedure is tantamount to approximating the thermodynamics of real gas mixtures by means of Amagat's Law. Newtonian iteration schemes for equilibrating the pressures and temperatures and computing the resulting approximate state relations of the mixture have been described by Cranfill ${ }^{3,4}$ and Cook. ${ }^{1}$

Alternative approaches or "mixing rules" have sometimes been contemplated but have generally been found less satisfactory. In particular, the use of Dalton's Law rather than Amagat's Law to approximate the thermodynamic state relations of the mixture is simpler because it requires no pressure equilibration, but quantum molecular dynamics simulations have shown that it is generally less accurate as well, except at low densities and/or high temperatures. ${ }^{5-7}$ This is not surprising, since Dalton's Law greatly underestimates interparticle interaction energies in dense systems, which is the essential reason why it is generally less accurate than Amagat's Law for real gases.

The partitioning and apportioning procedure based on pressure and temperature equilibration provides a tractable and intuitively appealing approximation to the true state relations of the mixture. Unfortunately, that approximation is uncontrolled; i.e., its accuracy cannot readily be quantified or systematically improved. However, its appeal and credibility are considerably enhanced by the fact that it correctly reproduces the exact state relations in mixtures of neutral (non-ionized) ideal gases. ${ }^{8}$ This highly desirable property is unfortunately lost in partially ionized gases, but it can be restored by equilibrating the chemical potentials, partial pressures, or number densities of the free electrons instead of the total pressures. ${ }^{8}$ The fact that the latter equilibration is exact for ideal gases suggests that it is likely to be more accurate in dense systems as well, and this has been confirmed by orbital-free molecular dynamics (OFMD) simulations $^{9}$ and theoretical calculations based on a free-energy minimization model. ${ }^{10}$

Whatever quantities are equilibrated, the resulting equations are highly nonlinear and must in general be solved by iterative methods. For this purpose, the algorithm described in Ref. 1 can be applied as it stands to enforce pressure and temperature equilibration in single-temperature plasma mixtures, but it requires generalization to equilibrate quantities other than the pressure, and to accommodate two-temperature plasmas in which the free electrons and heavy particles have different temperatures. The purpose of this paper is to present a generalized algorithm which provides both of those additional features. The present formulation is accordingly developed in 
a general form that provides the option to equilibrate an arbitrary independent thermodynamic variable $q$ instead of the total pressure $p$. A conventional pressure equilibration is regained simply by setting $q=p$, so that it becomes a simple matter to compare the resulting state relations with those obtained by alternative choices for $q$. The other choices of greatest interest are likely to be the free electron number density $n_{e}$, pressure $p_{e}$, and chemical potential $\mu_{e}{ }^{8}$ all of which are equivalent for ideal gases, but not in general. If the average degree of ionization $Z_{k}^{*}$ of material $k$ is known, its free electron number density is simply $n_{e k}=Z_{k}^{*} n_{k}$, where $n_{k}$ is the number density of the heavy particles (atoms and ions) of material $k$.

We remark parenthetically that although the present discussion is focused on homogeneous mixtures in which the constituents are intimately mixed on the molecular level, the numerical algorithm is equally applicable to heterogeneous or multiphase mixtures in which the different components actually do physically occupy distinct macroscopic spatial regions. In that case, pressure and temperature are ordinarily the appropriate physical quantities to equilibrate. Of course, those equilibrations are not really instantaneous but rather occur via physical processes with finite relaxation times. ${ }^{11,12}$ However, they can nevertheless be regarded as effectively instantaneous when their associated relaxation times are much shorter than the other characteristic physical time scales in the problem, and in that case the present method can profitably be employed. Conversely, nearly or effectively instantaneous pressure equilibration has sometimes been modeled as an artificial relaxation process that occurs over several time steps (see Ref. 12, and references cited therein), which is much easier to implement numerically but may introduce an unphysical dependence on the time step into the results.

Two-temperature plasmas correspondingly possess two internal energies as well, namely the specific internal energies $E_{i}$ and $E_{e}$ of the heavy particles and electrons, respectively, both of which we define as energies per unit total mass of the mixture, not per unit mass of the heavy particles or electrons. It should be noted that these energies are not purely thermal but also include "cold energy" (i.e., chemical/ionization energies or heats of formation), and hence do not in general vanish at zero temperature. In many hydrodynamic calculations, the internal energies are determined by energy transport equations, so $E_{i}$ and $E_{e}$ constitute independent thermodynamic variables which may be regarded as known quantities for present purposes. The algorithm presented in this paper is specifically designed for, and is restricted to, calculations of this type. In practice, however, one normally solves a transport equation for the total specific internal energy $E \equiv E_{i}+E_{e}$ rather than $E_{i}$, because $E$ satisfies a simpler equation than $E_{i}$ does (e.g., Ref. 13). One then simply obtains $E_{i}$ as $E_{i}=E-E_{e}$.

The other independent thermodynamic variables obtained by solving transport equations, which may therefore also be regarded as known quantities, are the mass density $\rho$ of the mixture, or equivalently its specific volume $\xi=1 / \rho$, and the mass fractions $Y_{k}$ of its constituent species or components, which of course must satisfy the constraint $\sum_{k} Y_{k}=1$. The artificial partitioning procedure described above then requires that the quantities $\xi, E_{i}$, and $E_{e}$ be apportioned among the different materials $k$ in such a way as to accomplish the desired equilibration. In the present context, of course, the heavy particles and electrons possess different temperatures $T_{i}$ and $T_{e}$, which must be separately and independently equilibrated among the different materials. We emphasize that this equilibration is a purely numerical process which bears no relation to, and should not be confused with, the physical tendency of $T_{i}$ and $T_{e}$ to equilibrate with each other on a time scale determined by the rate of energy exchange between heavy particles and electrons. ${ }^{13-19}$ Accurate theoretical expressions for that exchange rate, and the associated relaxation time it implies, are essential ingredients in the evolution equations for $E_{i}$ and $E_{e}$, but are irrelevant for present purposes.

The equations that must be solved to implement the procedure described above are of two types: constraint conditions to ensure that the specific volumes and internal energies of the materials are consistent with the known specific volume and energies of the mixture, and equilibration conditions that express and ensure the equilibration of $q, T_{i}$, and $T_{e}$ among the different materials in the mixture. These conditions are summarized in Secs. II and III, respectively. Together they constitute a closed nonlinear algebraic equation system, which must in general be solved by iterative methods. The Newtonian iteration scheme we use for this purpose is a natural generalization of that described in Ref. 1 and is presented in Sec. IV. Thermodynamic relations needed to evaluate various partial derivatives for the individual materials and the mixture as a whole in terms of known quantities are given in Secs. V and VI, respectively. The sound speed in the mixture requires special consideration and is derived in Sec. VII. Test calculations that illustrate iteration convergence rates as well as the different results obtained by equilibrating total pressure vs. free electron pressure are presented in Sec. VIII. A brief summary is given in Sec. IX.

\section{CONSTRAINT CONDITIONS}

For the most part, we shall use the same notation as Ref. 1 , the main exception being that specific internal energies are denoted by the symbol $E$ rather than $e$ so that $e$ is available for use as a subscript to refer to the electrons. The specific volume of material $k$ in the artificially partitioned mixture is denoted by $\xi_{k}$, and is defined as the volume occupied by material $k$ per unit mass of material $k$. Thus, $\rho_{k} \equiv$ $1 / \xi_{k}$ is the mass density of material $k$ within its subvolume. The mass of material $k$ per unit total volume is $Y_{k} \rho=Y_{k} / \xi$, so the volume fraction of material $k$ in the artificially partitioned mixture is $v_{k}=\xi_{k} Y_{k} / \xi$. These volume fractions must of course sum to unity, so that the quantities $\xi_{k}$ must satisfy the constraint

$$
\sum_{k=1}^{N} Y_{k} \xi_{k}=\xi
$$

where $N$ is the number of materials in the mixture.

The specific internal energies of the heavy particles and electrons of material $k$ within its subvolume are denoted by $E_{i k}$ and $E_{e k}$, respectively, both of which are defined per unit 
total mass of material $k$. The corresponding internal energies of the heavy particles and electrons of material $k$ per unit mass of the mixture are then simply $Y_{k} E_{i k}$ and $Y_{k} E_{e k}$, respectively. These quantities must clearly sum to $E_{i}$ and $E_{e}$, respectively, so that $E_{i k}$ and $E_{e k}$ must satisfy the constraints

$$
\begin{aligned}
& \sum_{k=1}^{N} Y_{k} E_{i k}=E_{i}, \\
& \sum_{k=1}^{N} Y_{k} E_{e k}=E_{e} .
\end{aligned}
$$

\section{EQUILIBRATION CONDITIONS}

Equations of state for each pure material $k$ are required as input to determine approximate state relations for the mixture, and are presumed to be available. Many if not most of the material state routines or packages in common use express the various thermodynamic variables of material $k$ as functions of the independent variables $\left(\rho_{k}, T_{i k}, T_{e k}\right)$. Normally, $E_{i k}\left(\rho_{k}, T_{i k}, T_{e k}\right)$ is independent of $T_{e k}$ and $E_{e k}\left(\rho_{k}, T_{i k}, T_{e k}\right)$ is independent of $T_{i k}$, but we shall retain those dependencies for generality. The functional relations $E_{i k}\left(\rho_{k}, T_{i k}, T_{e k}\right)$ and $E_{e k}\left(\rho_{k}, T_{i k}, T_{e k}\right)$ can in principle be inverted to obtain $T_{i k}\left(\rho_{k}, E_{i k}, E_{e k}\right)$ and $T_{e k}\left(\rho_{k}, E_{i k}, E_{e k}\right)$. It is generally assumed that the total pressure $p_{k}$ of material $k$ is given by the sum of the partial pressures $p_{i k}$ and $p_{e k}$ of its heavy particles and free electrons, respectively, so that $p_{k}=p_{i k}\left(\rho_{k}, T_{i k}, T_{e k}\right)+p_{e k}\left(\rho_{k}, T_{i k}, T_{e k}\right)$. The latter functional relations for $p_{i k}$ and $p_{e k}$ can be combined with those for $T_{i k}$ and $T_{e k}$ to obtain $p_{i k}\left(\rho_{k}, E_{i k}, E_{e k}\right)$ and $p_{e k}\left(\rho_{k}, E_{i k}, E_{e k}\right)$. Normally, $p_{i k}$ is independent of $T_{e k}$ or $E_{e k}$ and $p_{e k}$ is independent of $T_{i k}$ or $E_{i k}$, but we shall again retain those dependencies for generality.

It is essential to note that state routines or packages developed for practical applications often consider the excitation energy of bound electrons in excited states to be included in $E_{e k}$ rather than $E_{i k}$. The physical basis for that convention is that it simplifies the state relations when the excited state populations are primarily determined by $T_{e}$ rather than $T_{i}$, as is often assumed to be the case. ${ }^{20,21}$ That convention is not presumed in the present development, which is generally valid regardless of the physical interpretations of $E_{i k}$ and $E_{e k}$, provided they are the same for all materials $k$. However, consistency obviously requires the mixture energies $E_{i}$ and $E_{e}$ to adhere to the same convention as $E_{i k}$ and $E_{e k}$, which in turn requires that the transport equation for $E_{e}$ be formulated in a manner corresponding to the definition of $E_{e k}$ on which the state relations are based. In other words, if the excitation energy of bound electrons in excited states is included in the quantities $E_{e k}$, that energy must likewise be included in $E_{e}$ and the transport equation that determines it.

In accordance with the discussion of Sec. I, the equilibration conditions that we impose to implicitly determine the quantities $\xi_{k}\left(=1 / \rho_{k}\right), E_{i k}$, and $E_{e k}$ are as follows:

$$
\begin{gathered}
q_{k}\left(\rho_{k}, T_{i}, T_{e}\right)=q, \\
T_{i k}\left(\rho_{k}, E_{i k}, E_{e k}\right)=T_{i},
\end{gathered}
$$

$$
T_{e k}\left(\rho_{k}, E_{i k}, E_{e k}\right)=T_{e}
$$

Equations (1)-(6) constitute a system of $3 N+3$ equations in the $3 N+3$ unknown quantities $\xi_{k}, E_{i k}, E_{e k}, q, T_{i}$, and $T_{e}$. This system is highly nonlinear and must in general be solved by iterative methods. A suitable iteration scheme for this purpose is described in the next section.

\section{NEWTONIAN ITERATION SCHEME}

Let $\eta$ be the iteration index, which will be displayed as a superscript. Thus, the approximate value of any quantity $Q$ after iteration $\eta$ but before iteration $\eta+1$ is denoted by $Q^{\eta}$. The iteration scheme defines how the quantities $\xi_{k}, E_{i k}, E_{e k}$, $q, T_{i}$, and $T_{e}$ are advanced from iteration $\eta$ to iteration $\eta+1$. This will be done by means of linearized approximations to Eqs. (1)-(6). The constraint Eqs. (1)-(3) are already linear, so they become

$$
\begin{aligned}
& \sum_{k=1}^{N} Y_{k} \xi_{k}^{\eta+1}=\xi, \\
& \sum_{k=1}^{N} Y_{k} E_{i k}^{\eta+1}=E_{i}, \\
& \sum_{k=1}^{N} Y_{k} E_{e k}^{\eta+1}=E_{e} .
\end{aligned}
$$

The remainder of the scheme is defined by writing linearized approximations for the changes in $\xi_{k}, E_{i k}$, and $E_{e k}$ from iteration $\eta$ to iteration $\eta+1$ required to produce values of $q_{k}^{\eta+1}, T_{i k}^{\eta+1}$, and $T_{e k}^{\eta+1}$ that satisfy Eqs. (4)-(6):

$$
\begin{aligned}
\xi_{k}^{\eta+1}-\xi_{k}^{\eta}= & \left(\frac{\partial \xi_{k}}{\partial q_{k}}\right)\left(q^{\eta+1}-q_{k}^{\eta}\right)+\left(\frac{\partial \xi_{k}}{\partial T_{i k}}\right)\left(T_{i}^{\eta+1}-T_{i k}^{\eta}\right) \\
& +\left(\frac{\partial \xi_{k}}{\partial T_{e k}}\right)\left(T_{e}^{\eta+1}-T_{e k}^{\eta}\right), \\
E_{i k}^{\eta+1}-E_{i k}^{\eta}= & \left(\frac{\partial E_{i k}}{\partial q_{k}}\right)\left(q^{\eta+1}-q_{k}^{\eta}\right)+\left(\frac{\partial E_{i k}}{\partial T_{i k}}\right)\left(T_{i}^{\eta+1}-T_{i k}^{\eta}\right) \\
& +\left(\frac{\partial E_{i k}}{\partial T_{e k}}\right)\left(T_{e}^{\eta+1}-T_{e k}^{\eta}\right), \\
E_{e k}^{\eta+1}-E_{e k}^{\eta}= & \left(\frac{\partial E_{e k}}{\partial q_{k}}\right)\left(q^{\eta+1}-q_{k}^{\eta}\right)+\left(\frac{\partial E_{e k}}{\partial T_{i k}}\right)\left(T_{i}^{\eta+1}-T_{i k}^{\eta}\right) \\
& +\left(\frac{\partial E_{e k}}{\partial T_{e k}}\right)\left(T_{e}^{\eta+1}-T_{e k}^{\eta}\right),
\end{aligned}
$$

where it is understood that partial derivatives with respect to any of the variables $\left(q_{k}, T_{i k}, T_{e k}\right)$ are taken with the other two held constant and are evaluated at iteration $\eta$. Since all quantities are presumed known at the previous iteration $\eta$, Eqs. (7)-(12) constitute a determinate system of $3 N+3$ equations in the $3 N+3$ unknown quantities $\xi_{k}^{\eta+1}, E_{i k}^{\eta+1}, E_{e k}^{\eta+1}$, $q^{\eta+1}, T_{i}^{\eta+1}$, and $T_{e}^{\eta+1}$. It is apparent by inspection that if the iteration converges as $\eta \rightarrow \infty$, it produces a solution of Eqs. (1)-(6).

Substituting Eqs. (10)-(12) into Eqs. (7)-(9), we obtain 


$$
\begin{aligned}
& A_{q q} q^{\eta+1}+A_{q i} T_{i}^{\eta+1}+A_{q e} T_{e}^{\eta+1}=B_{q}, \\
& A_{i q} q^{\eta+1}+A_{i i} T_{i}^{\eta+1}+A_{i e} T_{e}^{\eta+1}=B_{i}, \\
& A_{e q} q^{\eta+1}+A_{e i} T_{i}^{\eta+1}+A_{e e} T_{e}^{\eta+1}=B_{e}
\end{aligned}
$$

where

$$
\begin{aligned}
A_{q q} & =\sum_{k} Y_{k}\left(\frac{\partial \xi_{k}}{\partial q_{k}}\right) ; \quad A_{q i}=\sum_{k} Y_{k}\left(\frac{\partial \xi_{k}}{\partial T_{i k}}\right) ; \\
A_{q e} & =\sum_{k} Y_{k}\left(\frac{\partial \xi_{k}}{\partial T_{e k}}\right), \\
A_{i q} & =\sum_{k} Y_{k}\left(\frac{\partial E_{i k}}{\partial q_{k}}\right) ; \quad A_{i i}=\sum_{k} Y_{k}\left(\frac{\partial E_{i k}}{\partial T_{i k}}\right) ; \\
A_{i e} & =\sum_{k} Y_{k}\left(\frac{\partial E_{i k}}{\partial T_{e k}}\right), \\
A_{e q} & =\sum_{k} Y_{k}\left(\frac{\partial E_{e k}}{\partial q_{k}}\right) ; \quad A_{e i}=\sum_{k} Y_{k}\left(\frac{\partial E_{e k}}{\partial T_{i k}}\right) ; \\
A_{e e} & =\sum_{k} Y_{k}\left(\frac{\partial E_{e k}}{\partial T_{e k}}\right), \\
B_{q}= & \xi-\sum_{k} Y_{k} \xi_{k}^{\eta} \\
& +\sum_{k} Y_{k}\left[q_{k}^{\eta}\left(\frac{\partial \xi_{k}}{\partial q_{k}}\right)+T_{i k}^{\eta}\left(\frac{\partial \xi_{k}}{\partial T_{i k}}\right)+T_{e k}^{\eta}\left(\frac{\partial \xi_{k}}{\partial T_{e k}}\right)\right], \\
B_{i}= & E_{i}-\sum_{k} Y_{k} E_{i k}^{\eta} \\
& +\sum_{k} Y_{k}\left[q_{k}^{\eta}\left(\frac{\partial E_{i k}}{\partial q_{k}}\right)+T_{i k}^{\eta}\left(\frac{\partial E_{i k}}{\partial T_{i k}}\right)+T_{e k}^{\eta}\left(\frac{\partial E_{i k}}{\partial T_{e k}}\right)\right], \\
B_{e}= & E_{e}-\sum_{k} Y_{k} E_{e k}^{\eta} Y_{k}\left[q_{k}^{\eta}\left(\frac{\partial E_{e k}}{\partial q_{k}}\right)+T_{i k}^{\eta}\left(\frac{\partial E_{e k}}{\partial T_{i k}}\right)+T_{e k}^{\eta}\left(\frac{\partial E_{e k}}{\partial T_{e k}}\right)\right] .
\end{aligned}
$$

The thermodynamic identities needed to evaluate the partial derivatives in Eqs. (16)-(21) in terms of derivatives with respect to $\left(\rho_{k}, T_{i k}, T_{e k}\right)$ are summarized in Sec. V.

Equations (13)-(15) are a system of three linear equations in the three unknown quantities $q^{\eta+1}, T_{i}^{\eta+1}$, and $T_{e}^{\eta+1}$, the solution to which is readily obtained from Cramer's rule. To minimize multiplications, it is convenient to compute the minors of the matrix $A$ at the outset and save them for repeated use. These quantities are given by

$$
\begin{aligned}
& M_{q q}=A_{i i} A_{e e}-A_{e i} A_{i e}, \\
& M_{i q}=A_{q i} A_{e e}-A_{e i} A_{q e}, \\
& M_{e q}=A_{q i} A_{i e}-A_{i i} A_{q e}, \\
& M_{q i}=A_{i q} A_{e e}-A_{e q} A_{i e}, \\
& M_{i i}=A_{q q} A_{e e}-A_{e q} A_{q e},
\end{aligned}
$$

$$
\begin{aligned}
& M_{e i}=A_{q q} A_{i e}-A_{i q} A_{q e}, \\
& M_{q e}=A_{i q} A_{e i}-A_{e q} A_{i i}, \\
& M_{i e}=A_{q q} A_{e i}-A_{e q} A_{q i}, \\
& M_{e e}=A_{q q} A_{i i}-A_{i q} A_{q i} .
\end{aligned}
$$

According to Cramer's rule, the solution of Eqs. (13)-(15) is then given by

$$
\begin{gathered}
q^{\eta+1}=R\left(B_{q} M_{q q}-B_{i} M_{i q}+B_{e} M_{e q}\right), \\
T_{i}^{\eta+1}=R\left(-B_{q} M_{q i}+B_{i} M_{i i}-B_{e} M_{e i}\right), \\
T_{e}^{\eta+1}=R\left(B_{q} M_{q e}-B_{i} M_{i e}+B_{e} M_{e e}\right),
\end{gathered}
$$

where

$$
R=1 /\left(A_{q q} M_{q q}-A_{q i} M_{q i}+A_{q e} M_{q e}\right) .
$$

Equations (31)-(33) combine with Eqs. (10)-(12) to provide explicit expressions for $\xi_{k}^{\eta+1}, E_{i k}^{\eta+1}$, and $E_{e k}^{\eta+1}$, which in turn determine $q_{k}^{\eta+1}, T_{i k}^{\eta+1}$, and $T_{e k}^{\eta+1}$ via the individual material state relations.

Newtonian iteration schemes are notoriously vulnerable to overshoots, so limiters are sometimes necessary to ensure convergence. Experience in applying the above iteration scheme on a variety of problems has shown that divergence is usually avoided if $\xi_{k}^{\eta+1}$ is not allowed to differ from $\xi_{k}^{\eta}$ by more than a factor of two. Limiters could also be applied to $E_{i k}^{\eta+1}$ and $E_{e k}^{\eta+1}$, but this has not been found necessary except when the initial guesses are very far off.

The iteration procedure is normally initialized by setting $\xi_{k}^{0}, E_{i k}^{0}$, and $E_{e k}^{0}$ equal to their values from the previous time step. Note that there is no need to initialize $q, T_{i}$, or $T_{e}$ since Eqs. (10)-(12) do not involve $q^{\eta}, T_{i}^{\eta}$, or $T_{e}^{\eta}$. The iteration is continued until $\max \left(\epsilon_{q k}^{\eta}, \epsilon_{i k}^{\eta}, \epsilon_{e k}^{\eta}\right)<\epsilon$ for all $k$, where $\epsilon \approx 10^{-4}$ and

$$
\begin{aligned}
\epsilon_{q k}^{\eta} & \equiv\left|Y_{k}\left(1-q_{k}^{\eta} / q^{\eta}\right)\right|, \\
\epsilon_{i k}^{\eta} & \equiv\left|Y_{k}\left(1-T_{i k}^{\eta} / T_{i}^{\eta}\right)\right|, \\
\epsilon_{e k}^{\eta} & \equiv\left|Y_{k}\left(1-T_{e k}^{\eta} / T_{e}^{\eta}\right)\right| .
\end{aligned}
$$

Once the above convergence criteria have been satisfied, the final converged values of $q, T_{i}$, and $T_{e}$ define the values of those thermodynamic quantities for the mixture. When $q \neq p$, the final converged material pressures $p_{k}\left(\rho_{k}, T_{i}, T_{e}\right)$ remain unequal, and the total pressure of the mixture is then given by their volume-weighted average; ${ }^{8}$ i.e.,

$$
p=\sum_{k} v_{k} p_{k}=\rho \sum_{k} \xi_{k} Y_{k} p_{k} .
$$

\section{THERMODYNAMIC DERIVATIVES FOR THE INDIVIDUAL MATERIALS}

The following thermodynamic identities can be derived by the usual straightforward but tedious manipulations. ${ }^{22}$ They express the partial derivatives of any thermodynamic 
variable $F_{k}$ of material $k$ (in particular, $F_{k}=\xi_{k}, E_{i k}$, and $E_{e k}$ ) with respect to $\left(q_{k}, T_{i k}, T_{e k}\right)$ in terms of derivatives with respect to the variables $\left(\rho_{k}, T_{i k}, T_{e k}\right)$, which are the conventional independent thermodynamic variables normally used to construct tabulated state relations. The relations below may then be used to evaluate the derivatives in Eqs. (10)-(12) and (16)-(21).

$$
\begin{gathered}
\left(\frac{\partial F_{k}}{\partial q_{k}}\right)_{T}=\left(\frac{\partial F_{k}}{\partial \rho_{k}}\right)_{T}\left(\frac{\partial q_{k}}{\partial \rho_{k}}\right)_{T}^{-1}, \\
\left(\frac{\partial F_{k}}{\partial T_{i k}}\right)_{q}=\left(\frac{\partial F_{k}}{\partial T_{i k}}\right)_{\rho}-\left(\frac{\partial F_{k}}{\partial \rho_{k}}\right)_{T}\left(\frac{\partial q_{k}}{\partial T_{i k}}\right)_{\rho}\left(\frac{\partial q_{k}}{\partial \rho_{k}}\right)_{T}^{-1}, \\
\left(\frac{\partial F_{k}}{\partial T_{e k}}\right)_{q}=\left(\frac{\partial F_{k}}{\partial T_{e k}}\right)_{\rho}-\left(\frac{\partial F_{k}}{\partial \rho_{k}}\right)_{T}\left(\frac{\partial q_{k}}{\partial T_{e k}}\right)_{\rho}\left(\frac{\partial q_{k}}{\partial \rho_{k}}\right)_{T}^{-1},
\end{gathered}
$$

where a subscript $q$ indicates that $q_{k}$ is held constant, a subscript $T$ indicates that both $T_{i k}$ and $T_{e k}$ are held constant, and it is understood that partial derivatives with respect to either $T_{i k}$ or $T_{e k}$ are always taken with the other held constant, even though this is not explicitly indicated by a subscript.

\section{THERMODYNAMIC DERIVATIVES FOR THE MIXTURE}

The iteration scheme determines the mixture state relations for the dependent thermodynamic variables $\left(q, T_{i}, T_{e}\right)$ as functions of the independent thermodynamic variables $\left(\rho, E_{i}, E_{e}\right)$. To obtain the mixture specific heats, and for various other purposes, it is necessary to evaluate partial derivatives of the mixture state relations. This may be done by taking the differentials of the constraint conditions of Eqs. (1)-(3) and the equilibration conditions of Eqs. (4)-(6) with respect to $\left(q, T_{i}, T_{e}\right)$ and combining the results to obtain

$$
\begin{gathered}
d \xi=A_{q q} d q+A_{q i} d T_{i}+A_{q e} d T_{e}, \\
d E_{i}=A_{i q} d q+A_{i i} d T_{i}+A_{i e} d T_{e}, \\
d E_{e}=A_{e q} d q+A_{e i} d T_{i}+A_{e e} d T_{e},
\end{gathered}
$$

where the quantities $A_{a b}(a, b=q, i, e)$ are the final converged values of the matrix elements defined in Eqs. (16)-(18). Equations (42)-(44) are a linear system of the same form as Eqs. (13)-(15), with $\left(B_{q}, B_{i}, B_{e}\right)$ replaced by $\left(d \xi, d E_{i}, d E_{e}\right)$ and $\left(q, T_{i}, T_{e}\right)^{\eta+1}$ replaced by $\left(d q, d T_{i}, d T_{e}\right)$. It then follows from Eqs. (31) to (33) that

$$
\begin{gathered}
d q=R\left(M_{q q} d \xi-M_{i q} d E_{i}+M_{e q} d E_{e},\right. \\
d T_{i}=R\left(-M_{q i} d \xi+M_{i i} d E_{i}-M_{e i} d E_{e}\right), \\
d T_{e}=R\left(M_{q e} d \xi-M_{i e} d E_{i}+M_{e e} d E_{e}\right) .
\end{gathered}
$$

The partial derivatives of $\left(E_{i}, E_{e}\right)$ with respect to $\left(\rho, T_{i}, T_{e}\right)$ can be determined by regarding Eqs. (46) and (47) as a system of two equations for $d E_{i}$ and $d E_{e}$, the solution of which is readily found to be

$$
d E_{i}=J\left[R\left(M_{e e} M_{q i}-M_{e i} M_{q e}\right) d \xi+M_{e e} d T_{i}+M_{e i} d T_{e}\right],
$$

$$
d E_{e}=J\left[R\left(M_{i e} M_{q i}-M_{i i} M_{q e}\right) d \xi+M_{i e} d T_{i}+M_{i i} d T_{e}\right]
$$

where $1 / J=R\left(M_{i i} M_{e e}-M_{i e} M_{e i}\right)$. Since $\xi=1 / \rho$, it follows at once that

$$
\begin{gathered}
\left(\frac{\partial E_{i}}{\partial \rho}\right)_{T}=\left(R J / \rho^{2}\right)\left(M_{e i} M_{q e}-M_{e e} M_{q i}\right), \\
\left(\frac{\partial E_{i}}{\partial T_{i}}\right)_{\rho}=J M_{e e} ; \quad\left(\frac{\partial E_{i}}{\partial T_{e}}\right)_{\rho}=J M_{e i}, \\
\left(\frac{\partial E_{e}}{\partial \rho}\right)_{T}=\left(R J / \rho^{2}\right)\left(M_{i i} M_{q e}-M_{i e} M_{q i}\right), \\
\left(\frac{\partial E_{e}}{\partial T_{i}}\right)_{\rho}=J M_{i e} ; \quad\left(\frac{\partial E_{e}}{\partial T_{e}}\right)_{\rho}=J M_{i i},
\end{gathered}
$$

where as before a subscript $T$ indicates that both $T_{i}$ and $T_{e}$ are held constant, and it is understood that partial derivatives with respect to $T_{i}$ or $T_{e}$ are always taken with the other held constant, even though this is not explicitly indicated by a subscript.

The partial derivatives of the mixture pressure must be evaluated from Eq. (38), which implies

$$
d p=\rho \sum_{k} Y_{k}\left[\xi_{k} d p_{k}+\left(p_{k}-p\right) d \xi_{k}\right]
$$

It follows that

$$
\begin{aligned}
& \left(\frac{\partial p}{\partial q}\right)_{T}=\rho \sum_{k} Y_{k}\left[\xi_{k}\left(\frac{\partial p_{k}}{\partial q_{k}}\right)+\left(p_{k}-p\right)\left(\frac{\partial \xi_{k}}{\partial q_{k}}\right)\right], \\
& \left(\frac{\partial p}{\partial T_{i}}\right)_{q}=\rho \sum_{k} Y_{k}\left[\xi_{k}\left(\frac{\partial p_{k}}{\partial T_{i k}}\right)+\left(p_{k}-p\right)\left(\frac{\partial \xi_{k}}{\partial T_{i k}}\right)\right], \\
& \left(\frac{\partial p}{\partial T_{e}}\right)_{q}=\rho \sum_{k} Y_{k}\left[\xi_{k}\left(\frac{\partial p_{k}}{\partial T_{e k}}\right)+\left(p_{k}-p\right)\left(\frac{\partial \xi_{k}}{\partial T_{e k}}\right)\right],
\end{aligned}
$$

where it is again understood that partial derivatives with respect to any of the independent variables $\left(q_{k}, T_{i k}, T_{e k}\right)$ are taken with the other two held constant. The partial derivatives of $p_{k}$ and $\xi_{k}$ with respect to $\left(q_{k}, T_{i k}, T_{e k}\right)$ can be expressed in terms of more conventional derivatives with respect to $\left(\rho_{k}, T_{i k}, T_{e k}\right)$ by setting $F_{k}=p_{k}$ and $F_{k}=\xi_{k}$ in Eqs. (39)-(41). Once this has been done and the derivatives of $p$ with respect to $\left(q, T_{i}, T_{e}\right)$ have been evaluated by means of Eqs. (55)-(57), they can be converted into derivatives with respect to the more conventional independent variables $\left(\rho, T_{i}, T_{e}\right)$ by means of the following further thermodynamic identities:

$$
\begin{gathered}
\left(\frac{\partial p}{\partial \rho}\right)_{T}=\left(\frac{\partial p}{\partial q}\right)_{T}\left(\frac{\partial q}{\partial \rho}\right)_{T} \\
\left(\frac{\partial p}{\partial T_{i}}\right)_{\rho}=\left(\frac{\partial p}{\partial T_{i}}\right)_{q}+\left(\frac{\partial p}{\partial q}\right)_{T}\left(\frac{\partial q}{\partial T_{i}}\right)_{\rho} \\
\left(\frac{\partial p}{\partial T_{e}}\right)_{\rho}=\left(\frac{\partial p}{\partial T_{e}}\right)_{q}+\left(\frac{\partial p}{\partial q}\right)_{T}\left(\frac{\partial q}{\partial T_{e}}\right)_{\rho}
\end{gathered}
$$


in which the derivatives of $q$ with respect to $\left(\rho, T_{i}, T_{e}\right)$ follow immediately from Eq. (42) and are given by

$$
\begin{aligned}
& \left(\frac{\partial q}{\partial \rho}\right)_{T}=-\frac{1}{\rho^{2} A_{q q}} \\
& \left(\frac{\partial q}{\partial T_{i}}\right)_{\rho}=-\frac{A_{q i}}{A_{q q}} \\
& \left(\frac{\partial q}{\partial T_{e}}\right)_{\rho}=-\frac{A_{q e}}{A_{q q}} .
\end{aligned}
$$

\section{THE SOUND SPEED}

In this section, we derive the sound speed of the mixture in the limit of slow energy exchange between heavy particles and electrons. The usual thermodynamic expressions for sound speed are not directly applicable in two-temperature plasmas, where there are three rather than two independent thermodynamic variables. In this situation, the simplest way to proceed is to go back to basics and directly determine the sound speed by linearizing the two-temperature hydrodynamic equations about a uniform steady state. We shall restrict attention to the case in which the rate of energy exchange between heavy particles and electrons is very slow compared to the other physical time scales in the problem, in particular those associated with the propagation of sound waves. Under these conditions, the relation between acoustic variations in pressure and density can be inferred from the adiabatic thermodynamic relations

$$
\begin{gathered}
d p=K_{\rho} d \rho+K_{i} d E_{i}+K_{e} d E_{e}, \\
d E_{i}=\frac{p_{i}}{\rho^{2}} d \rho, \\
d E_{e}=\frac{p_{e}}{\rho^{2}} d \rho,
\end{gathered}
$$

where $p_{e}=\sum_{k} v_{k} p_{e k}$ is the partial pressure of the free electrons, $p_{i}=p-p_{e}$, and

$$
\begin{aligned}
& K_{\rho}=\left(\frac{\partial p}{\partial \rho}\right)_{E}, \\
& K_{i}=\left(\frac{\partial p}{\partial E_{i}}\right)_{\rho}, \\
& K_{e}=\left(\frac{\partial p}{\partial E_{e}}\right)_{\rho},
\end{aligned}
$$

where a subscript $E$ indicates that both $E_{i}$ and $E_{e}$ are held constant, and it is understood that partial derivatives with respect to either $E_{i}$ or $E_{e}$ are always taken with the other held constant, even though this is not explicitly indicated by a subscript. The electron pressures $p_{e k}$ are determined as functions of $\left(\rho_{k}, T_{i}, T_{e}\right)$ by the thermodynamic state relations of the individual materials $k$. Combining Eqs. (64)-(66), we obtain

$$
d p=c^{2} d \rho
$$

where

$$
\rho^{2} c^{2}=\rho^{2} K_{\rho}+p_{i} K_{i}+p_{e} K_{e}
$$

The linearized hydrodynamic equations in one spatial dimension $x$ reduce to

$$
\begin{aligned}
& \frac{\partial \rho}{\partial t}=-\rho \frac{\partial u}{\partial x}, \\
& \rho \frac{\partial u}{\partial t}=-\frac{\partial p}{\partial x},
\end{aligned}
$$

where $u$ is the fluid velocity. Combining Eqs. (70), (72), and (73), we obtain

$$
\frac{\partial^{2} \rho}{\partial t^{2}}=c^{2} \frac{\partial^{2} \rho}{\partial x^{2}},
$$

which is just the familiar wave equation in one dimension, thereby confirming that $c$ as determined by Eq. (71) is indeed the sound speed.

The derivatives $K_{\rho}, K_{i}$, and $K_{e}$ can be evaluated by means of still further thermodynamic identities

$$
\begin{aligned}
K_{\rho} & =\left(\frac{\partial p}{\partial \rho}\right)_{E} \\
& =\left(\frac{\partial p}{\partial \rho}\right)_{T}+\left(\frac{\partial p}{\partial T_{i}}\right)_{\rho}\left(\frac{\partial T_{i}}{\partial \rho}\right)_{E}+\left(\frac{\partial p}{\partial T_{e}}\right)_{\rho}\left(\frac{\partial T_{e}}{\partial \rho}\right)_{E}, \\
K_{i} & =\left(\frac{\partial p}{\partial E_{i}}\right)_{\rho}=\left(\frac{\partial p}{\partial T_{i}}\right)_{\rho}\left(\frac{\partial T_{i}}{\partial E_{i}}\right)_{\rho}+\left(\frac{\partial p}{\partial T_{e}}\right)_{\rho}\left(\frac{\partial T_{e}}{\partial E_{i}}\right)_{\rho}, \\
K_{e} & =\left(\frac{\partial p}{\partial E_{e}}\right)_{\rho}=\left(\frac{\partial p}{\partial T_{i}}\right)_{\rho}\left(\frac{\partial T_{i}}{\partial E_{e}}\right)_{\rho}+\left(\frac{\partial p}{\partial T_{e}}\right)_{\rho}\left(\frac{\partial T_{e}}{\partial E_{e}}\right)_{\rho}
\end{aligned}
$$

in which the partial derivatives of $p$ with respect to $\left(\rho, T_{i}, T_{e}\right)$ were determined in Eqs. (58)-(60), while those of $\left(T_{i}, T_{e}\right)$ with respect to $\left(\rho, E_{i}, E_{e}\right)$ follow immediately from Eqs. (46) and (47) and are given by

$$
\begin{aligned}
& \left(\frac{\partial T_{i}}{\partial \rho}\right)_{E}=\frac{R M_{q i}}{\rho^{2}} ; \quad\left(\frac{\partial T_{i}}{\partial E_{i}}\right)_{\rho}=R M_{i i} ; \quad\left(\frac{\partial T_{i}}{\partial E_{e}}\right)_{\rho}=-R M_{e i}, \\
& \left(\frac{\partial T_{e}}{\partial \rho}\right)_{E}=-\frac{R M_{q e}}{\rho^{2}} ; \quad\left(\frac{\partial T_{e}}{\partial E_{i}}\right)_{\rho}=-R M_{i e} ; \quad\left(\frac{\partial T_{e}}{\partial E_{e}}\right)_{\rho}=R M_{e e} .
\end{aligned}
$$

\section{TEST CALCULATIONS}

In this section, we report the results of test calculations that illustrate the convergence behavior of the iteration algorithm and the differences that result from equilibrating electron pressure rather than total pressure. State relations for the pure individual materials in these calculations were obtained from the widely used LEOS package, ${ }^{23}$ which is based on the QEOS $^{24}$ and HQEOS ${ }^{25}$ models and Thomas-Fermi theory. In general, the electron pressure consists of a thermal part, which vanishes by definition at $T_{e}=0$, and a "cold" part, which vanishes in classical ideal gases but can be 

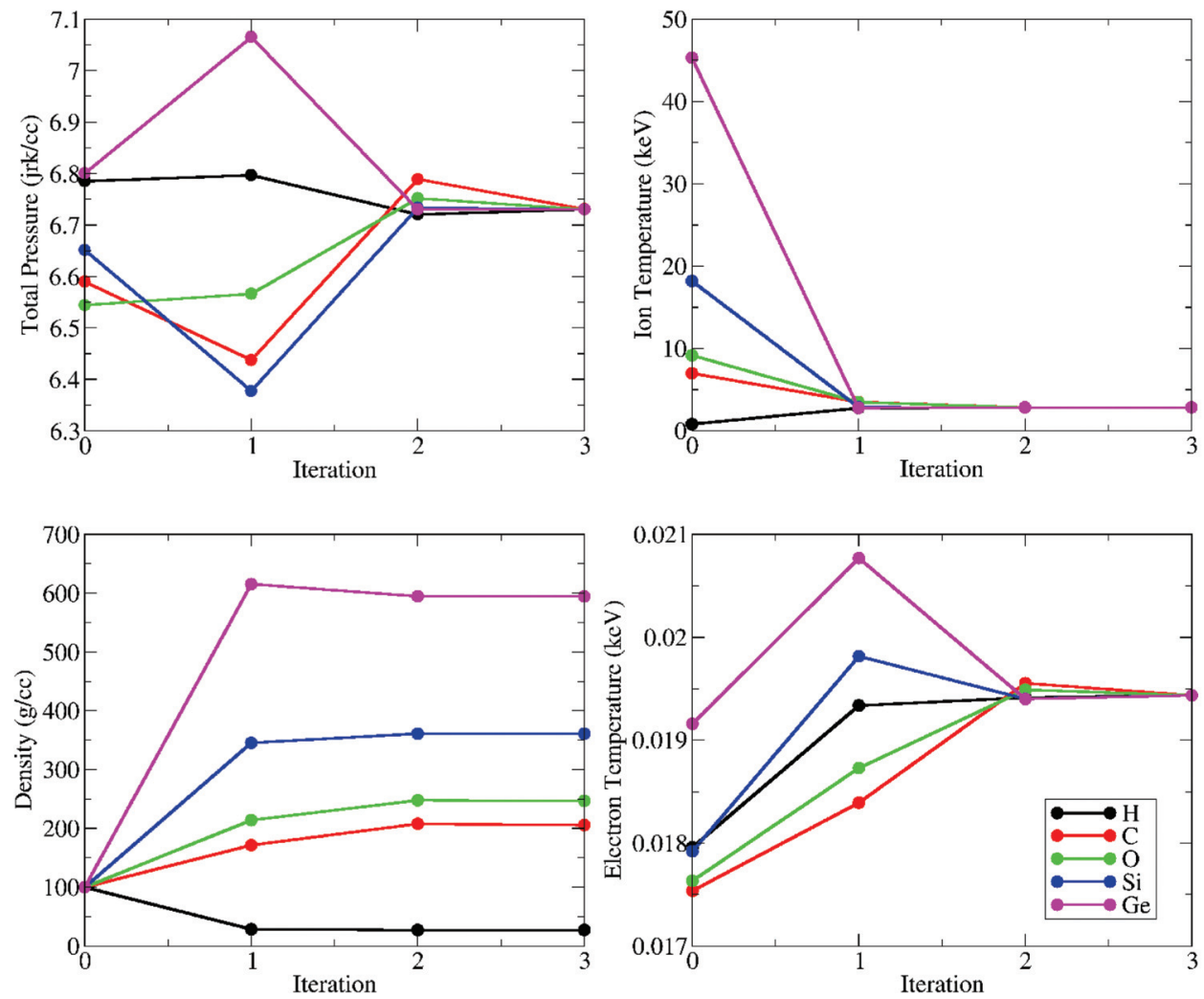

FIG. 1. Convergence of species densities, total pressures, ion temperatures, and electron temperatures during the course of Newton-Raphson iteration. significant in dense and/or degenerate systems. LEOS computes both parts internally and includes them in the total pressure, but unfortunately provides only the thermal part of $p_{e}$ to the user. For best results in practical applications, the "cold" part of $p_{e}$ should be computed and added to the thermal part prior to equilibrating $p_{e}$. This was not done in the present test calculations, which are merely intended to be illustrative, so the present results for $p_{e}$ equilibration were obtained by equilibrating the thermal contributions only.

We performed test calculations for three different mixtures. The first is a mixture of materials commonly used in inertial confinement fusion capsules, namely hydrogen $(\mathrm{H})$, carbon $(\mathrm{C})$, oxygen $(\mathrm{O})$, silicon $(\mathrm{Si})$, and germanium $(\mathrm{Ge})$. The mass fractions of these five elements are equal, so that
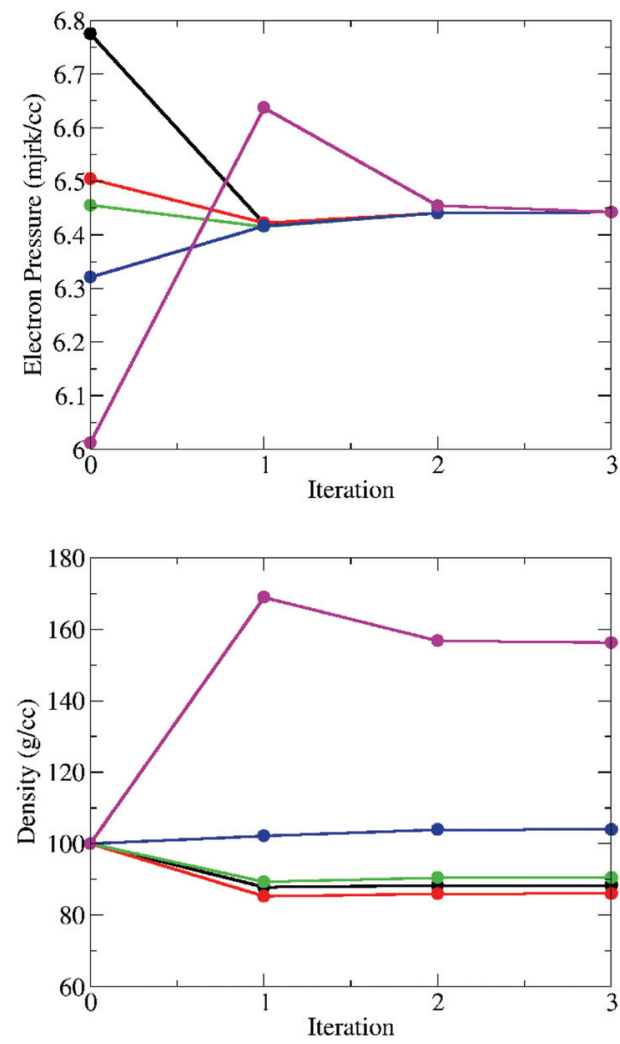
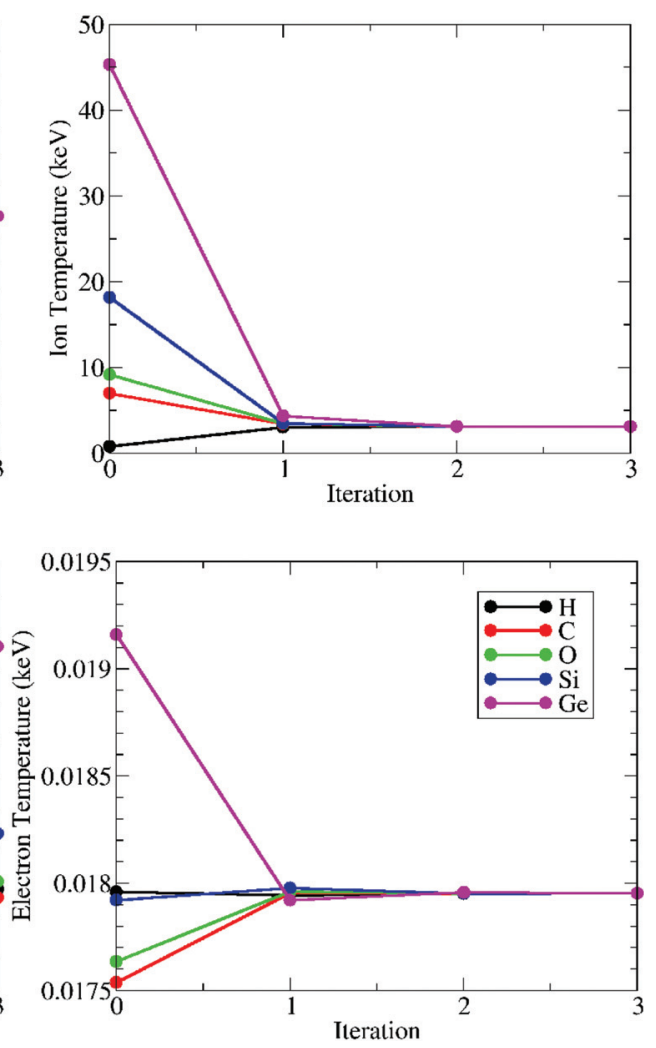

FIG. 2. Convergence of species densities, electron pressures, ion temperatures, and electron temperatures during the course of Newton-Raphson iteration. 
TABLE I. Differences between equilibrating $p$ and $p_{e}$ in $\mathrm{H}-\mathrm{C}-\mathrm{O}-\mathrm{Si}-\mathrm{Ge}$ mixture.

\begin{tabular}{lcccccc}
\hline \hline & $p(\mathrm{Mbar})$ & $p_{e}(\mathrm{Mbar})$ & $T_{i}(\mathrm{eV})$ & $T_{e}(\mathrm{eV})$ & $c(\mathrm{~cm} / \mu \mathrm{s})$ & Iterations \\
\hline$p$ equil. & 67306.42 & 64.67 & 2840.53 & 19.44 & 33.53 & 3 \\
$p_{e}$ equil. & 66669.25 & 64.42 & 3134.39 & 17.95 & 33.26 & 3 \\
\hline \hline
\end{tabular}

$Y_{k}=0.2$ for $k=1, \cdots, 5$. The density, total energy, and electron energy of the mixture are $\rho=100 \mathrm{~g} / \mathrm{cc}, E=10^{15} \mathrm{erg} / \mathrm{g}$, and $E_{e}=10^{12} \mathrm{erg} / \mathrm{g}$. These conditions correspond to a very wide separation between the ion and electron temperatures, with the ions much hotter than the electrons. The iteration is initialized by setting the starting guesses for the species densities and energies to the mixture values; i.e., $\rho_{k}=\rho, E_{i k}=$ $E-E_{e}$ and $E_{e k}=E_{e}$. The convergence of the iteration procedure is shown in Fig. 1 for total pressure equilibration, and in Fig. 2 for electron pressure equilibration. In both cases, convergence requires only three iterations, in spite of the large changes in species densities and ion temperatures. The final converged values are seen to be significantly different in the two cases, especially the species densities, which are much closer together when $p_{e}$ is equilibrated instead of $p$. However, it is noteworthy that the sound speed nevertheless differs by less than $1 \%$ between the two cases, as shown in Table I.

The second mixture considered was polystyrene (i.e., an equimolar mixture of carbon and hydrogen), under conditions where the electron temperature exceeds the ion temperature. As before, we set the starting guesses for the species densities and energies to the mixture values: $\rho_{C}=\rho_{H}=\rho=2.0 \mathrm{~g} / \mathrm{cc}$, $E_{C}=E_{H}=E=0.0926 \mathrm{jrk} / \mathrm{g}$ and $E_{e C}=E_{e H}=E_{e}=0.08 \mathrm{jrk} / \mathrm{g}$. Table II summarizes the results obtained by equilibrating both $p$ and $p_{e}$. The resulting total pressures differ by less than $0.1 \%$, which reflects the fact that $p_{e}$ constitutes about $85 \%$ of $p$, so that there is little difference between the two equilibrations under these conditions. Also shown are the corresponding LEOS results for the $\mathrm{CH}$ mixture, which predict a slightly higher $p$, possibly because they include the "cold" part of $p_{e}$ omitted in our calculations.

The third mixture considered was an equimolar mixture of $\mathrm{He}$ and $\mathrm{Fe}$ previously simulated by Lambert et al. ${ }^{9}$ using OFMD. We performed calculations corresponding to a mixture density and temperature of $\rho=1 \mathrm{~g} / \mathrm{cc}$ and $T=T_{i}=T_{e}=500 \mathrm{eV}$, for which their OFMD results for pressure are given in the second row of their Table II. Since our algorithm uses energies rather than temperatures as independent mixture variables, we specified mixture energies of $E=Y_{H e}$ $E_{\mathrm{He}}+Y_{\mathrm{Fe}} E_{\mathrm{Fe}}=0.04958115139 \mathrm{jrk} / \mathrm{g}$ and $E_{e}=Y_{\mathrm{He}} E_{e \mathrm{He}}+Y_{\mathrm{Fe}}$ $E_{e F e}=0.04708882090 \mathrm{jrk} / \mathrm{g}$ to obtain temperatures that closely

TABLE II. Differences between equilibrating $p$ and $p_{e}$ in C-H mixture.

\begin{tabular}{lcccccc}
\hline \hline & $p(\mathrm{Mbar})$ & $p_{e}(\mathrm{Mbar})$ & $T_{i}(\mathrm{eV})$ & $T_{e}(\mathrm{eV})$ & $c(\mathrm{~cm} / \mu \mathrm{s})$ & iterations \\
\hline$p$ equil. & 1143.08 & 978.45 & 528.22 & 966.54 & 30.82 & 3 \\
$p_{e}$ equil. & 1142.12 & 977.97 & 527.37 & 965.82 & 30.79 & 3 \\
LEOS CH table & 1151.63 & 977.97 & 527.85 & 965.73 & 30.89 & $\mathrm{NA}$ \\
\hline \hline
\end{tabular}

TABLE III. Differences between equilibrating $p$ and $p_{e}$ in He-Fe mixture.

\begin{tabular}{lcccccc}
\hline \hline & $p$ (Mbar) & $p_{e}(\mathrm{Mbar})$ & $T_{i}(\mathrm{eV})$ & $T_{e}(\mathrm{eV})$ & $c(\mathrm{~cm} / \mu \mathrm{s})$ & iterations \\
\hline$p$ equil. & 203.835 & 186.367 & 503.737 & 502.389 & 17.632 & 4 \\
$p_{e}$ equil. & 203.482 & 185.938 & 501.557 & 500.762 & 17.584 & 3 \\
OFMD $^{9}$ & 200.06 & $\ldots$ & 500 & 500 & $\ldots$ & $\mathrm{NA}$ \\
\hline \hline
\end{tabular}

approximate $500 \mathrm{eV}$. The starting guesses for the densities and energies of the individual materials are again the mixture values: $\rho_{\mathrm{He}}=\rho_{\mathrm{Fe}}=\rho, E_{\mathrm{He}}=E_{\mathrm{Fe}}=E$ and $E_{\text {eHe }}=E_{e F e}=E_{e}$. Table III shows our results for total pressure equilibration and electron pressure equilibration, together with the total pressure predicted by the OFMD simulations of Lambert et al. There is no significant difference between the total pressures we obtain by equilibrating $p$ vs. $p_{e}$, both of which are about $2 \%$ higher than the OFMD value.

None of these calculations and comparisons provides direct evidence that equilibrating $p_{e}$ produces physically more accurate results than equilibrating $p$, which would require including the "cold" part of $p_{e}$. It further requires accurate simulations of the state relations for both the true mixture and the individual pure materials by the same method, as was done by Lambert et al. ${ }^{9}$ Those authors compared their mixture simulations with the results of equilibrating $p$ vs. the excess pressure $P_{e x}$ defined in their Eq. (4), and found that the latter produced more accurate results. Note that $p_{e}$ becomes identical to $P_{e x}$ when the heavy particles are treated as a classical ideal gas, so that $P_{e x}$ may be regarded as an approximation to $p_{e}$.

Other tests show that the method typically converges rapidly at high temperatures, where thermodynamic derivatives are smooth, but can run into trouble at low temperatures where $\partial p_{k} / \partial \rho_{k}$ can pass through zero (e.g., phase changes), and $p_{e}$ or $E_{e}$ and their derivatives go to zero and/or become unreliable due to deficiencies in the electronic state relations when ionization is negligible (e.g., neutral gases). A workaround for these situations is to put floors $p_{0}, p_{e 0}$, and $E_{e 0}$ under $p_{k}, p_{e k}$, and $E_{e k}$ and their partial derivatives, such that $\partial p_{k} / \partial \rho_{k} \geq p_{0} / \rho_{k}, \partial p_{e k} / \partial \rho_{k} \geq$ $p_{e 0} / \rho_{k}$ and $\partial E_{e k} / \partial T_{e k} \geq E_{e 0} / T_{e k}$. This reduces the accuracy of the method at low temperatures, but has been found sufficient for problems with strong heating in which energies quickly increase by several orders of magnitude, thereby producing almost immediate ionization.

\section{SUMMARY}

We have presented an iterative algorithm for determining pressures and temperatures in a two-temperature plasma mixture as functions of density, total energy, electron energy, and the constituent mass fractions. Various partial derivatives of the mixture state relations, including the sound speed and specific heats, have also been derived and expressed in terms of known quantities. The algorithm is based on an equilibration procedure in which the temperatures and one additional thermodynamic variable, normally either the total pressure or the partial pressure of the free electrons, are 
equilibrated among the pure constituents. The algorithm has been applied to a variety of inertial confinement fusion problems and found to converge rapidly, provided the electron temperature is sufficiently high for significant ionization.

\section{ACKNOWLEDGMENTS}

This work was performed under the auspices of the U.S. Department of Energy by Lawrence Livermore National Laboratory under Contract No. DE-AC52-07NA27344. We are grateful to Bill Cabot, John Castor, C. H. Chang, Jeff Greenough, Rob Managan, Phil Sterne, Heather Whitley, Mark Ulitsky, and George Zimmerman for many helpful discussions, and to C. H. Chang for calling our attention to Refs. 3 and 4. This paper is dedicated to the memory of Charles W. Cranfill (April 24, 1945-April 15, 2013).

${ }^{1}$ A. W. Cook, "Enthalpy diffusion in multicomponent flows," Phys. Fluids 21, 055109 (2009).

${ }^{2}$ M. Gittings, R. Weaver, M. Clover, T. Betlach, N. Byrne, R. Coker, E. Dendy, R. Hueckstaedt, K. New, W. R. Oakes, R. Ranta, and D. Stefan, "The RAGE radiation-hydrodynamic code," Comput. Sci. Discovery 1, 015005 (2008).

${ }^{3} \mathrm{C}$. W. Cranfill, "MIXPAC: A subroutine package for calculating equations of state for equilibrium mixtures of materials," Los Alamos National Laboratory Report No. LA-9861-M, 1983.

${ }^{4} \mathrm{C}$. W. Cranfill, "EOS of a material mixture in pressure equilibrium," Los Alamos National Laboratory Report No. LA-13661, 2000.

${ }^{5}$ J. G. Clérouin, V. Recoules, S. Mazevet, P. Noiret, and P. Renaudin, "Experiments and simulations on Al-Au mixtures and mixtures laws," Phys. Rev. B 76, 064204 (2007).

${ }^{6}$ D. A. Horner, J. D. Kress, and L. A. Collins, "Quantum molecular dynamics simulations of warm dense lithium hydride: Examination of mixing rules," Phys. Rev. B 77, 064102 (2008).

${ }^{7}$ R. J. Magyar and T. R. Mattsson, "Mixing of equations of state for xenondeuterium using density functional theory," Phys. Plasmas 20, 032701 (2013).

${ }^{8}$ J. D. Ramshaw, "Approximate thermodynamic state relations in partially ionized gas mixtures,” Phys. Plasmas 11, 3572-3578 (2004).
${ }^{9}$ F. Lambert, J. Clérouin, J.-F. Danel, L. Kazandjian, and G. Zérah, "Direct verification of mixing rules in the hot and dense regime," Phys. Rev. E 77, $026402(2008)$

${ }^{10}$ G. Massacrier, A. Y. Potekhin, and G. Chabrier, "Equation of state for partially ionized carbon and oxygen mixtures at high temperatures," Phys. Rev. E 84, 056406 (2011).

${ }^{11}$ C. H. Chang and J. D. Ramshaw, "Dynamical evolution of volume fractions in multipressure multiphase flow models," Phys. Rev. E 77, 066305 (2008).

${ }^{12}$ Y. V. Yanilkin, E. A. Goncharov, V. Y. Kolobyanin, V. V. Sadchikov, J. R. Kamm, M. J. Shashkov, and W. J. Rider, "Multi-material pressure relaxation methods for lagrangian hydrodynamics," Comput. Fluids 83, 137-143 (2013).

${ }^{13}$ C. H. Chang and J. D. Ramshaw, "Numerical simulation of nonequilibrium effects in an argon plasma jet," Phys. Plasmas 1, 3698-3708 (1994).

${ }^{14} \mathrm{H}$. Brysk, "Electron-ion equilibration in a partially degenerate plasma," Plasma Phys. 16, 927-932 (1974).

${ }^{15}$ D. O. Gericke and M. S. Murillo, "Dense plasma temperature equilibration in the binary collision approximation," Phys. Rev. E 65, 036418 (2002).

${ }^{16}$ L. S. Brown, D. L. Preston, and R. L. Singleton, Jr., "Charged particle motion in a highly ionized plasma," Phys. Rep. 410, 237-333 (2005).

${ }^{17}$ L. S. Brown and R. L. Singleton, Jr., "Temperature equilibration rate with Fermi-Dirac statistics," Phys. Rev. E 76, 066404 (2007).

${ }^{18}$ J. Daligault and G. Dimonte, "Correlation effects on the temperaturerelaxation rates in dense plasmas," Phys. Rev. E 79, 056403 (2009).

${ }^{19}$ L. X. Benedict, M. P. Surh, J. I. Castor, S. A. Khairallah, H. D. Whitley, D. F. Richards, J. N. Glosli, M. S. Murillo, C. R. Scullard, P. E. Grabowski, D. Michta, and F. R. Graziani, "Molecular dynamics simulations and generalized Lenard-Balescu calculations of electron-ion temperature equilibration in plasmas," Phys. Rev. E 86, 046406 (2012).

${ }^{20}$ M. Mitchner and C. H. Kruger, Jr., Partially Ionized Gases (John Wiley and Sons, Inc., New York, N.Y., 1973).

${ }^{21}$ C. G. Braun and J. A. Kunc, "Collisional-radiative coefficients from a three-level atomic model in nonequilibrium argon plasmas," Phys. Fluids 30, 499-509 (1987).

${ }^{22}$ L. E. Reichl, A Modern Course in Statistical Physics (John Wiley and Sons, Inc., New York, N.Y., 1998).

${ }^{23}$ E. M. Corey and D. A. Young, AIP Conf. Proc. 429, $43-46$ (1998).

${ }^{24}$ R. H. More, K. H. Warren, D. A. Young, and G. B. Zimmerman, "A new quotidian equation of state (QES) for hot dense matter," Phys. Fluids 31, 3059-3078 (1988).

${ }^{25}$ D. A. Young and E. M. Corey, "A new global equation of state model for hot, dense matter,” J. Appl. Phys. 78, 3748-3755 (1995). 